\title{
Transformações do nitrogênio no solo após adição de dejeto líquido e cama sobreposta de suínos
}

\author{
Sandro José Giacomini(1), Celso Aita(1), Stefen Barbosa Pujol(1) e Ezequiel César Carvalho Miola(1) \\ (1)Universidade Federal de Santa Maria, Centro de Ciências Rurais, Departamento de Solos, Avenida Roraima, no 1.000, Camobi, \\ CEP 97105-900 Santa Maria, RS. E-mail: sjgiacomini@gmail.com, celsoaita@gmail.com, pujolstefen@gmail.com, ezequielmiola@gmail.com
}

Resumo - O objetivo deste trabalho foi avaliar as transformações do N no solo após a aplicação de dejeto líquido (DLS) e cama sobreposta (CSS) de suínos, com e sem palha de aveia, e com e sem incorporação ao solo. Os tratamentos avaliados foram: solo $(\mathrm{S}), \mathrm{S}+$ palha incorporada $(\mathrm{S}+\mathrm{Pi}), \mathrm{S}+$ palha em superfície $(\mathrm{S}+\mathrm{Ps}), \mathrm{S}+\mathrm{CSS}$ incorporada $(\mathrm{S}+\mathrm{CSSi}), \mathrm{S}+\mathrm{CSS}$ em superfície (S + CSSs), S + DLS incorporado (S + DLSi), S + DLS em superfície (S + DLSs), S + Pi + DLSi e S + Ps + DLSs. Avaliaram-se a volatilização de amônia, a nitrificação e a mineralização do N do DLS e da CSS. A incorporação do DLS reduziu a volatilização de amônia em 93\%, em comparação à aplicação na superfície do solo. O N amoniacal da CSS foi nitrificado nos cinco primeiros dias. A taxa de nitrificação líquida foi duas vezes maior com a incorporação do DLS ao solo do que na superfície. A mineralização do $\mathrm{N}$ orgânico não diferiu com o modo de aplicação do DLS e da CSS no solo. Na CSS, $14,6 \%$ do $\mathrm{N}$ foi mineralizado, contra $34,9 \%$ no DLS. A aplicação do DLS com a palha de aveia na superfície do solo favorece a imobilização microbiana de $\mathrm{N}$, mas não reduz as perdas de $\mathrm{N}$ por volatilização de amônia.

Termos para indexação: imobilização de $\mathrm{N}$, mineralização, nitrificação, volatilização de $\mathrm{NH}_{3}$.

\section{Nitrogen transformations in soil after the addition of pig slurry and pig deep-litter}

\begin{abstract}
The objective of this work was to evaluate soil $\mathrm{N}$ transformations after the application of pig slurry (PS) and pig deep-litter (PDL), with and without oat straw, and with and without incorporation into the soil. The evaluated treatments were: soil $(\mathrm{S}), \mathrm{S}+$ incorporated straw $(\mathrm{S}+\mathrm{Sti}), \mathrm{S}+$ straw on surface $(\mathrm{S}+\mathrm{Sts}), \mathrm{S}+$ incorporated PDL (S + PDLi), S + PDL on surface (S + PDLs), S + incorporated PS (S + PSi), $\mathrm{S}+\mathrm{PS}$ on surface $(\mathrm{S}+\mathrm{PSs}), \mathrm{S}+\mathrm{Sti}+\mathrm{Psi}$, and $\mathrm{S}+\mathrm{Sts}+\mathrm{PSs}$. The following were evaluated: ammonia volatilization, nitrification and $\mathrm{N}$ mineralization of PS and PDL. Incorporation of PS reduced ammonia volatilization in $93 \%$ when compared to surface application. The ammoniacal $\mathrm{N}$ in PDL was nitrified within the first five days. The net nitrification rate was two times higher with the incorporation of PS into the soil than at surface. Organic N mineralization did not differ with the application methods for PS and PDL to the soil. In PDL, $14.6 \% \mathrm{~N}$ was mineralized, against $34.9 \%$ in PS. The application of PS with oat straw to soil surface favors microbial $\mathrm{N}$ immobilization but it does not reduce $\mathrm{N}$ losses by ammonia volatilization.
\end{abstract}

Index terms: $\mathrm{N}$ imobilization, mineralization, nitrification, $\mathrm{NH}_{3}$ volatilization.

\section{Introdução}

O nitrogênio é o nutriente encontrado em maior concentração nos dejetos de suínos (Sánchez \& González, 2005), e a proporção entre N orgânico e mineral depende, principalmente, do manejo dos dejetos adotado na criação dos animais. O dejeto de suíno manejado na forma líquida (DLS) e armazenado em esterqueira anaeróbica se caracteriza pelo baixo teor de matéria seca e pela elevada percentagem de $\mathrm{N}$ amoniacal (40 a $70 \%$ do $\mathrm{N}$ total) (Sánchez \& González, 2005). Já o dejeto de suíno manejado no sistema de cama sobreposta (CSS) apresenta elevado teor de matéria seca e elevada proporção de $\mathrm{N}$ na forma orgânica (70 a 90\% do $\mathrm{N}$ total) (Oliveira, 1999). Essas diferenças podem afetar a dinâmica do $\mathrm{N}$ após a adição desses dois tipos de dejetos de suínos no solo. No DLS, a disponibilidade desse nutriente será condicionada pelo destino da fração amoniacal (Giacomini \& Aita, 2008), enquanto no caso da CSS, ela dependerá da taxa de mineralização do N orgânico.

Após a aplicação do DLS ao solo, podem ocorrer perdas significativas de $\mathrm{N}$ por volatilização de $\mathrm{NH}_{3}$, e a intensidade desse processo é influenciada pelas condições ambientais, pelas características físicas e 
químicas do DLS, e pelo modo de aplicação ao solo (Dendooven et al., 1998; Port et al., 2003). Na maioria das situações, a aplicação de DLS na superfície do solo resulta em maiores perdas de $\mathrm{N}$ por volatilização de $\mathrm{NH}_{3}$ do que quando este dejeto é incorporado (Dendooven et al., 1998). Nos últimos anos, é cada vez mais comum a aplicação de DLS na superfície do solo, em sistema plantio direto, e, na primavera/verão, a prática é aplicar o DLS sobre resíduos culturais de trigo e aveia (Port et al., 2003). A mistura do DLS, rico em $\mathrm{N}$ amoniacal, com a palha, cuja relação $\mathrm{C} / \mathrm{N}$ é normalmente elevada, pode resultar na imobilização de $\mathrm{N}$ do dejeto pela biomassa microbiana durante a decomposição da palha (Recous et al., 1995; Giacomini et al., 2009). A intensidade desse processo terá reflexos sobre a quantidade de $\mathrm{N}$ volatilizado, pois quanto maior for a imobilização logo após a aplicação do DLS, menor será a quantidade de $\mathrm{N}$ que estará sujeita à volatilização. Além disso, a imobilização competirá com o processo de nitrificação, uma vez que o $\mathrm{N}$ amoniacal imobilizado não estará disponível para as bactérias nitrificadoras. Essa possível competição entre a imobilização e a nitrificação pode reduzir a quantidade de $\mathrm{NO}_{3}^{-}$no solo, gerado a partir da nitrificação do $\mathrm{N}$ amoniacal dos dejetos, o que pode ter implicações no potencial fertilizante e poluente desse material orgânico. Isso porque, na maioria das propriedades que utilizam o DLS como fonte de nutrientes para as plantas, a sua aplicação é realizada antes da semeadura das culturas. Nessa condição, o N é aplicado ao solo em uma fase em que a demanda por esse nutriente é nula. Assim, o aumento da imobilização de $\mathrm{NH}_{4}^{+}$, aplicado com os dejetos nessa fase, poderá resultar em menor quantidade de $\mathrm{NO}_{3}{ }^{-}$no solo, o que reduz o potencial de perda de $\mathrm{N}$ por lixiviação e desnitrificação. Contudo, são escassos os estudos que envolvem a avaliação simultânea dos processos de imobilização e nitrificação do $\mathrm{N}$ amoniacal aplicado com o DLS no solo.

Também não foram encontrados trabalhos sobre a nitrificação e a mineralização do $\mathrm{N}$ aplicado com a CSS no solo. A mineralização do $\mathrm{N}$ desse material orgânico dependerá das condições edafoclimáticas após sua aplicação no solo e, também, da sua composição química (Morvan \& Nicolardot, 2009), a qual varia conforme o substrato utilizado na formação da CSS e do seu tempo de permanência na pocilga (Oliveira, 1999). Os substratos mais comumente utilizados para formar a cama são a maravalha e a serragem (Oliveira, 1999), materiais caracterizados pela elevada relação $\mathrm{C} / \mathrm{N}$ e pela concentração de compostos lignificados. Quanto maior o tempo de permanência da CSS na pocilga, maior deverá ser a proporção de $\mathrm{N}$ recalcitrante, haja vista a decomposição parcialmente aeróbia, à qual a cama é submetida, e a perda de $\mathrm{N}$ na forma gasosa, com volatilização e desnitrificação (Tiquia et al., 2002). Essas características são indicativas de que a mineralização do $\mathrm{N}$ orgânico adicionado ao solo pela CSS deverá ocorrer de forma lenta e gradual, diferentemente de quando o DLS é adicionado ao solo, em que a maior parte do $\mathrm{N}$ já se encontra na forma mineral (Sánchez \& González, 2005). O conhecimento da cinética e da quantidade de $\mathrm{N}$ mineralizado a partir da CSS é importante para a definição de doses e épocas de aplicação desse material orgânico ao solo como fonte de $\mathrm{N}$ às culturas.

O objetivo deste trabalho foi avaliar as transformações do N no solo após a aplicação de DLS e CSS de suínos, com e sem palha de aveia, e com e sem incorporação ao solo.

\section{Material e Métodos}

Os experimentos foram realizados no Laboratório de Biotransformações de Carbono e Nitrogênio (Labcen) da Universidade Federal de Santa Maria (UFSM), Rio Grande do Sul. O solo utilizado foi um Argissolo Vermelho distrófico arênico (Santos et al., 2006), coletado na camada de $0-10 \mathrm{~cm}$ de uma área que vinha sendo cultivada em sistema plantio direto há mais de dez anos. Após a coleta, o solo foi homogeneizado, peneirado em malha de $4 \mathrm{~mm}$ e armazenado úmido em sacos de plástico, em temperatura ambiente, por 13 dias até o momento da incubação. No momento da coleta, o solo apresentava $150 \mathrm{~g} \mathrm{~kg}^{-1}$ de argila; $\mathrm{pH}$ $\left(\mathrm{H}_{2} \mathrm{O}\right)$ de 5,2; índice SMP de 6,5; 16,2 $\mathrm{mg} \mathrm{L}^{-1}$ de $\mathrm{P}$; $46 \mathrm{mg} \mathrm{L}^{-1}$ de $\mathrm{K} ; 0,1 \mathrm{cmol}_{\mathrm{c}} \mathrm{L}^{-1}$ de $\mathrm{Al}^{3+} ; 2,5 \mathrm{cmol}_{\mathrm{c}} \mathrm{L}^{-1}$ de $\mathrm{Ca}^{2+} ; 0,6$ cmol $_{\mathrm{c}} \mathrm{L}^{-1}$ de $\mathrm{Mg}^{2+} ; 2,3$ cmol $_{\mathrm{c}} \mathrm{L}^{-1} \mathrm{de} \mathrm{H}+\mathrm{Al}^{3+}$; 3,3 cmol $_{\mathrm{c}} \mathrm{L}^{-1}$ de CTC efetiva; saturação de $\mathrm{Al}$ de 4,5\%; saturação por bases (T) de 58\%; e $18 \mathrm{~g} \mathrm{~kg}^{-1}$ de matéria orgânica.

A palha de aveia-preta foi coletada quando a planta estava no estádio de maturação fisiológica. Após a secagem ao ar, os grãos foram descartados, e os colmos e as folhas foram cortados em pedaços de 1 a $2 \mathrm{~cm}$. Uma subamostra da palha foi colocada em estufa, a $65^{\circ} \mathrm{C}$, para se obter a massa de matéria seca $\left(\mathrm{MS}, \mathrm{g} \mathrm{kg}^{-1}\right)$ 
e calcular a quantidade de palha seca ao ar $\left(\mathrm{mg} \mathrm{kg}^{-1}\right)$ a ser adicionada ao solo. A palha seca foi moída em moinho Willey equipado com peneira de malha 40 e analisada quanto aos teores de $\mathrm{N}$ total e $\mathrm{C}$ orgânico conforme Tedesco et al. (1995).

Os dejetos de suínos foram obtidos em dois sistemas distintos de criação dos animais. O DLS foi coletado em esterqueira anaeróbica de uma unidade com animais de maternidade e recria, enquanto a CSS foi coletada após terem sido criados cinco lotes de suínos de terminação (15 meses) sobre cama de maravalha. A MS dos dejetos foi determinada após a secagem destes em estufa, a $65^{\circ} \mathrm{C}$, até se obter massa constante. No material seco e moído, foram determinados os teores de C orgânico (Tedesco et al., 1995). No DLS, os teores de $\mathrm{N}$ total e $\mathrm{N}$ amoniacal $\left(\mathrm{NH}_{4}{ }^{+}+\mathrm{NH}_{3}\right)$ foram determinados de acordo com Aita et al. (2007), sem secagem prévia. Na CSS úmida, os teores de $\mathrm{N}$ total, $\mathrm{N}$ amoniacal e $\mathrm{N}$ nítrico $\left(\mathrm{NO}_{2}{ }^{-}+\mathrm{NO}_{3}{ }^{-}\right)$foram determinados em $1 \mathrm{~g}$ de material conforme Tedesco et al. (1995). A determinação do pH no DLS foi realizada diretamente em uma alíquota de aproximadamente $60 \mathrm{~mL}$ de dejetos e na CSS, o pH em água foi determinado com a mistura do material e água destilada na proporção de 1:1 (Tedesco et al., 1995). As características e as quantidades adicionadas de palha, DLS e CSS estão apresentadas na Tabela 1.

Utilizou-se o delineamento experimental inteiramente casualizado com quatro repetições. A mineralização do $\mathrm{N}$ foi avaliada nos tratamentos: $\mathrm{T} 1$, solo $(\mathrm{S})$; T2, $\mathrm{S}+$ palha incorporada $(\mathrm{S}+\mathrm{Pi})$; T3, S + palha em superfície $(\mathrm{S}+\mathrm{Ps})$; T4, S + CSS incorporada ( $\mathrm{S}+\mathrm{CSSi})$; T5, S + CSS em superfície (S + CSSs); T6, S + DLS incorporado (S + DLSi); T7, $\mathrm{S}+$ DLS em superfície (S + DLSs); T8, S + Pi + DLSi; e T9, S + Ps + DLSs. A volatilização de amônia foi avaliada somente nos tratamentos que receberam o DLS e no tratamento testemunha, sem a aplicação do dejeto. Os tratamentos foram acondicionados em incubadora na ausência de luz e com temperatura constante de $25^{\circ} \mathrm{C}$. Durante a incubação, a umidade do solo foi mantida na capacidade de campo, por meio da adição periódica de água.

Cada unidade experimental foi montada separadamente. Em recipientes cilíndricos de acrílico com $6,0 \mathrm{~cm}$ de altura e 5,3 cm de diâmetro interno, foram colocados 92,6 $\mathrm{g}$ de solo úmido (equivalente a $85 \mathrm{~g}$ de solo seco a $105^{\circ} \mathrm{C}$ ), cuja umidade foi elevada à capacidade de campo com adição de $7,1 \mathrm{~mL}$ de água ou de DLS. A altura final da camada de solo dentro de cada recipiente foi de aproximadamente $5 \mathrm{~cm}$. A forma como o solo foi umedecido variou conforme o tratamento. Nos tratamentos T3, T5, T7 e T9, adicionaram-se, em cada frasco de acrílico, 46,3 $\mathrm{g}$ de solo e, posteriormente, $3 \mathrm{~mL}$ de água. Em seguida, foram adicionados o restante do solo (46,3 g) e os materiais orgânicos, na superfície, conforme os diferentes tratamentos. Com exceção dos tratamentos com DLS (T6, T7, T8 e T9), nos demais tratamentos, foram aplicados 4,1 mL de água sobre os resíduos orgânicos para atingir a capacidade de campo. Nos tratamentos com DLS, o umedecimento do solo foi obtido por meio da fração líquida dos dejetos. Nos tratamentos com incorporação, o solo foi adicionado ao frasco de uma só vez $(92,6 \mathrm{~g})$ e, em seguida, foram adicionados 7,1 mL de água, nos tratamentos sem DLS (T2, T3, T4 e T5), e $3 \mathrm{~mL}$ de água nos tratamentos com DLS. A mistura do solo úmido com os materiais orgânicos foi feita manualmente com auxílio de espátula.

A quantidade de palha de aveia adicionada em cada frasco foi de 7,0 $\mathrm{g} \mathrm{kg}^{-1}$ de solo seco. Esta quantidade, ao

Tabela 1. Composição física e química, e quantidade adicionada dos dejetos líquidos de suínos, da cama sobreposta e da palha de aveia utilizados na incubação.

\begin{tabular}{|c|c|c|c|c|c|c|c|c|}
\hline Resíduo orgânico & $\mathrm{MS}^{(1)}$ & C orgânico & $\mathrm{N}$ total & $\mathrm{N}$ amoniacal & $\mathrm{N}$ nítrico & N orgânico & $\mathrm{C} / \mathrm{N}$ & $\mathrm{pH} \mathrm{H} \mathrm{H}_{2} \mathrm{O}$ \\
\hline \multicolumn{9}{|c|}{ Composição $\left(\mathrm{g} \mathrm{kg}^{-1}\right)$} \\
\hline Dejetos líquidos & 46 & 10 & 2,5 & 1,24 & - & 1,26 & 4,0 & 7,9 \\
\hline Cama sobreposta & 477 & 119 & 10,4 & 0,94 & 0,04 & 9,42 & 11,4 & 9,1 \\
\hline Palha de aveia & 890 & 432 & 9,3 & - & - & - & 46,5 & - \\
\hline \multicolumn{9}{|c|}{ Quantidade adicionada (mg kg-1 de solo seco) } \\
\hline Dejetos líquidos & 2.217 & 468 & 121,9 & 60,00 & - & 61,90 & - & - \\
\hline Cama sobreposta & 15.026 & 3.742 & 328,0 & 30,00 & 1,00 & 297,00 & - & - \\
\hline Palha de aveia & 7.000 & 3.025 & 65,0 & - & - & - & - & - \\
\hline
\end{tabular}

${ }^{(1)} \mathrm{MS}$, massa de matéria seca. 
se considerar a área do recipiente de acrílico, equivale a uma aplicação de $2,8 \mathrm{Mg} \mathrm{ha}^{-1}$ de palha. Antes da incubação, a palha foi umedecida com água destilada na proporção de $3 \mathrm{~mL} \mathrm{~g}^{-1}$ de palha. O DLS e a CSS foram adicionados nas quantidades de $48,2 \mathrm{~mL} \mathrm{~kg}^{-1}$ de solo seco $\left(20 \mathrm{~m}^{3} \mathrm{ha}^{-1}\right)$ e $31,5 \mathrm{~g} \mathrm{~kg}^{-1}$ de solo seco $\left(12,6 \mathrm{Mg} \mathrm{ha}^{-1}\right)$, respectivamente. No caso do DLS, a quantidade adicionada foi estabelecida para evitar a saturação do solo pela fração líquida deste, o que proporcionou uma adição de $\mathrm{N}$ mineral de aproximadamente $60 \mathrm{mg} \mathrm{kg}^{-1} \mathrm{de}$ solo que, segundo Recous et al. (1995), assegura uma condição não limitante de $\mathrm{N}$ à decomposição de palhas de cereais. A quantidade de CSS foi estabelecida para proporcionar uma adição próxima daquela realizada em condições de campo.

A volatilização de amônia foi avaliada apenas durante os primeiros cinco dias após a aplicação do DLS ao solo. Para tal, foi montado um sistema baseado no método proposto por Nömmik (1973), em que a amônia volatilizada foi captada em esponja embebida com solução de ácido fosfórico e glicerina, no interior dos frascos de vidro, e suspensa a $2 \mathrm{~cm}$ acima dos frascos de acrílico que continha os tratamentos. As trocas das esponjas foram realizadas aos 1, 2, 3 e 5 dias após a aplicação do DLS. Após cada coleta, as esponjas eram lavadas com $100 \mathrm{~mL}$ de $\mathrm{KCl} 1 \mathrm{~mol} \mathrm{~L}^{-1}$, e os teores de $\mathrm{N}_{-} \mathrm{NH}_{3}$ foram determinados por meio da destilação de uma alíquota de $20 \mathrm{~mL}$ dessa solução, após adição de 0,2 g de $\mathrm{MgO}$ (Tedesco et al., 1995).

No experimento destinado à avaliação da mineralização do $\mathrm{N}$, os teores de $\mathrm{N}$ mineral do solo $\left(\mathrm{N}-\mathrm{NH}_{4}{ }^{+}\right.$e $\left.\mathrm{N}^{-\mathrm{NO}_{2}}{ }^{-}+\mathrm{N}_{-} \mathrm{NO}_{3}{ }^{-}\right)$foram determinados logo após a incubação (T0) e aos 5, 10, 20, 30, 50 e 80 dias após o início da incubação. $\mathrm{O} \mathrm{N}$ mineral foi extraído de todo o solo incubado ( $85 \mathrm{~g}$ de solo seco), inclusive dos resíduos orgânicos, e determinado de acordo com Tedesco et al. (1995).

A nitrificação líquida diária do $\mathrm{N}$ amoniacal aplicado com o DLS e a CSS foi calculada a partir do acúmulo de $\mathrm{N}^{-\mathrm{NO}_{3}}{ }^{-}$no solo. Para isso, em cada

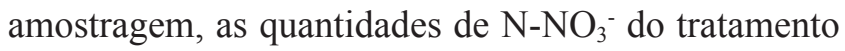
sem dejetos (S) foram descontadas das quantidades de $\mathrm{N}^{-\mathrm{NO}_{3}}{ }^{-}$encontradas nos tratamentos com dejetos (DLS e CSS), e o valor foi dividido pelo número de dias transcorridos entre as duas amostragens consideradas. O cálculo da taxa de nitrificação líquida foi feito apenas nas amostragens realizadas nos primeiros 20 dias. A mineralização líquida de $\mathrm{N}$ nos tratamentos foi estimada com base nos dados de $\mathrm{N}$ mineral medidos em cada data de avaliação, por meio da equação: $\mathrm{Nm}=(\mathrm{Nro} 2-\mathrm{Nro1})-(\mathrm{Ns} 2-\mathrm{Ns} 1)$, em que: $\mathrm{Nm}$ representa a quantidade $\left(\mathrm{mg} \mathrm{kg}^{-1}\right)$ de $\mathrm{N}$ mineralizado; Nro1 e Nro2 representam as quantidades de $\mathrm{N}$ mineral do solo nos tratamentos com resíduos orgânicos (P, DLS e CSS) no início e ao final de cada intervalo de avaliação, respectivamente; e Ns1 e Ns2 representam as quantidades de $\mathrm{N}$ mineral do solo no tratamento testemunha no início e ao final de cada intervalo de avaliação, respectivamente. Para o cálculo da mineralização líquida de $\mathrm{N}$ nos tratamentos T2 a T7, considerou-se, como testemunha, o tratamento T1. Para o cálculo nos tratamentos T8 e T9, foram utilizados, como testemunha, os tratamentos T6 e T7. Quando positivo, esse cálculo indica a ocorrência de mineralização líquida de $\mathrm{N}$ e quando negativo, imobilização líquida. A partir dos valores máximos de imobilização líquida de $\mathrm{N}$ nos tratamentos com palha de aveia, foi calculada a relação $\mathrm{N}$ imobilizado/C adicionado.

Os resultados obtidos para a volatilização de amônia, $\mathrm{N}$ mineral e mineralizado no solo foram submetidos à análise da variância, e, quando houve significância pelo teste $\mathrm{F}$, as médias de tratamentos foram comparadas pelo teste de Tukey, a 5\% de probabilidade.

\section{Resultados e Discussão}

Aincorporação do DLS ao soloreduziu a volatilização de amônia (Figura 1). Nos tratamentos em que o DLS foi aplicado na superfície do solo, a perda média de $\mathrm{N}$ por volatilização de $\mathrm{NH}_{3}$ em cinco dias foi de $11 \%$ (6,6 $\mathrm{mg} \mathrm{kg}^{-1}$ de $\mathrm{N}$ no solo) do $\mathrm{N}$ amoniacal aplicado; no entanto, nos tratamentos com incorporação, essa perda foi de apenas $0,8 \%\left(0,48 \mathrm{mg} \mathrm{kg}^{-1}\right.$ de $\mathrm{N}$ no solo $)$. Essa redução de $93 \%$ na volatilização de $\mathrm{NH}_{3}$ com a incorporação dos dejetos ao solo está de acordo com os resultados obtidos por Dendooven et al. (1998), que constataram, em condições de laboratório com a mesma temperatura $\left(25^{\circ} \mathrm{C}\right)$, que a incorporação do DLS ao solo reduziu a volatilização de amônia em $90 \%$, em comparação à aplicação dos dejetos na superfície. Com a aplicação dos dejetos sobre a palha de aveia, a perda de $\mathrm{N}$ por volatilização de amônia foi semelhante àquela ocorrida após a aplicação dos dejetos na superfície do solo, sem palha. Observou-se alta concentração de N amoniacal na superfície do solo, logo após a aplicação 
do DLS, que, aliada ao $\mathrm{pH}$ normalmente elevado dos dejetos de suínos (Tabela 1), pode aumentar a relação $\mathrm{NH}_{3} / \mathrm{NH}_{4}^{+}$e facilitar a emissão de $\mathrm{NH}_{3}$ para a atmosfera (Chantigny et al., 2004). Contudo, não foram encontrados trabalhos sobre o efeito da palha na volatilização de $\mathrm{NH}_{3}$ com o uso de DLS em condições de laboratório.

Quando o DLS foi aplicado na superfície do solo, a quase totalidade da perda de $\mathrm{NH}_{3}$, verificada durante os cinco dias de avaliação, ocorreu nos dois primeiros dias após a aplicação dos dejetos; no primeiro dia, foram emitidos $56 \%$ da quantidade total de amônia (Figura 1). Essa cinética de volatilização de $\mathrm{N}_{-} \mathrm{NH}_{3}$, com perdas mais acentuadas logo após a aplicação dos dejetos, foi relatada em trabalhos com dejetos de suínos, realizados em campo (Port et al., 2003; Chantigny et al., 2004).

A nitrificação do $\mathrm{N}$ amoniacal $\left(\mathrm{NH}_{3}+\mathrm{NH}_{4}^{+}\right)$aplicado ao solo com o DLS e com a CSS foi rápida (Figura 2). Constatou-se que todo o $\mathrm{N}$ amoniacal adicionado com a CSS foi nitrificado nos cinco primeiros dias, independentemente da forma de aplicação ao solo. Nos tratamentos com DLS, a nitrificação do $\mathrm{N}$ amoniacal foi mais lenta, tendo sido concluída nos dez primeiros dias após a incorporação (DLSi) e em 20 dias quando o dejeto foi aplicado na superfície (DLSs). Os resultados obtidos com os DLS são próximos aos

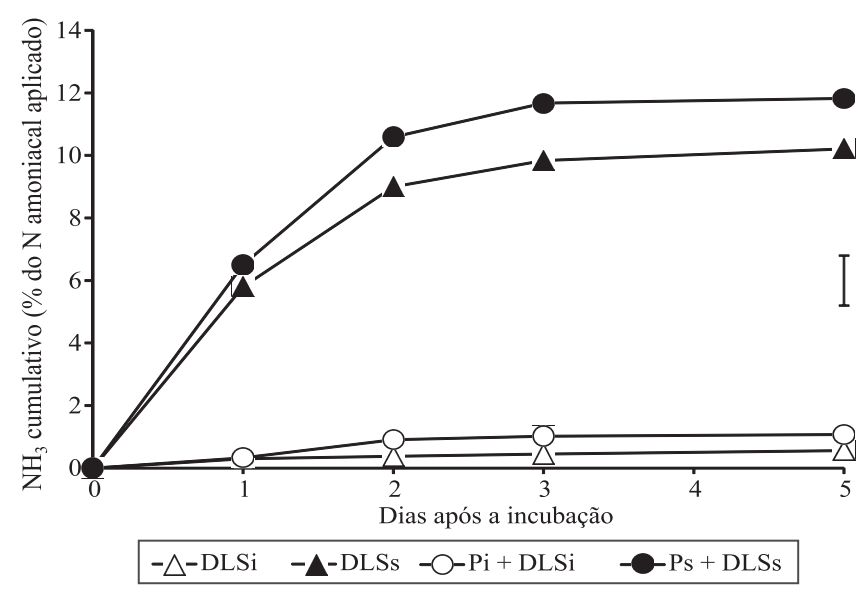

Figura 1. Volatilização acumulada de amônia nos tratamentos com adição de dejetos líquidos de suínos (DLS) e palha + dejetos líquidos $(\mathrm{P}+\mathrm{DLS})$ na superfície (s) ou incorporados (i) ao solo. A barra vertical indica a diferença mínima significativa pelo teste de Tukey, a 5\% de probabilidade. encontrados por Cordovil et al. (2011) em laboratório, a $24^{\circ} \mathrm{C}$, que observaram que o $\mathrm{N}$ amoniacal de DLS foi completamente nitrificado em 21 dias.

Os tratamentos CSSs e CSSi apresentaram, nos cinco primeiros dias, taxas de nitrificação de 5,3 e $4,8 \mathrm{mg} \mathrm{kg}^{-1}$ de $\mathrm{N}^{-\mathrm{NO}_{3}-}$ por dia, respectivamente. No mesmo período, as taxas de nitrificação no solo dos tratamentos com DLSs e DLSi foram semelhantes e não diferiram quando os dejetos foram aplicados isoladamente ou em conjunto com a palha, o que indica que a presença da palha não afetou a taxa de nitrificação do $\mathrm{N}$ amoniacal aplicado com os dejetos. Na média dos tratamentos com DLS, com e sem palha na superfície e incorporado, a taxa de nitrificação foi de 4,2 e $7,9 \mathrm{mg} \mathrm{kg}^{-1}$ de $\mathrm{N}^{-\mathrm{NO}_{3}}{ }^{-}$por dia, respectivamente. A taxa de nitrificação obtida com o DLSi foi aproximadamente o dobro daquela encontrada por Cordovil et al. (2011), os quais verificaram que o $\mathrm{N}^{-\mathrm{NH}_{4}}{ }^{+}$aplicado ao solo com o dejeto de suíno foi nitrificado a uma taxa de

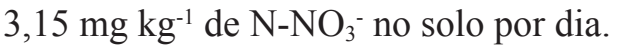

A redução na velocidade de nitrificação do $\mathrm{N}$ amoniacal ocorrida com a aplicação do dejeto líquido em superfície (DLSs), relativamente a sua incorporação (DLSi), pode contribuir para diminuir o potencial de perdas de $\mathrm{N}$ no sistema plantio direto, em que os dejetos são aplicados sobre os resíduos culturais. Os resultados obtidos são indicativos de que a incorporação do DLS ao solo pode reduzir as perdas de $\mathrm{N}$ por volatilização (Figura 1); porém, também pode provocar o rápido acúmulo de $\mathrm{NO}_{3}{ }^{-}$no solo após a aplicação do dejeto. Por isso, a importância de pesquisas para reduzir a taxa de nitrificação do $\mathrm{N}$ amoniacal dos dejetos, como, por exemplo, o uso de inibidores de nitrificação, um aspecto ainda pouco estudado no Brasil.

Com a aplicação do DLS e da CSS, tanto na presença como na ausência de palha, a quantidade de $\mathrm{N}$ mineral no solo aumentou significativamente desde a primeira amostragem, e esse aumento foi maior com o uso do DLS do que com a CSS (Figura 3). Na média dos quatro tratamentos com DLS, a quantidade de $\mathrm{N}$ mineral no solo da amostragem realizada logo após a aplicação dos tratamentos superou a do tratamento testemunha (S) em $66 \mathrm{mg} \mathrm{kg}^{-1}$, enquanto nos tratamentos com CSS, esse aumento foi de apenas $17 \mathrm{mg} \mathrm{kg}^{-1}$ de $\mathrm{N}$. Esses resultados são consequência das diferenças na composição entre os dois materiais orgânicos, em que o DLS apresentou maior concentração de $\mathrm{N}$ amoniacal do que a CSS (Tabela 1). 
No caso da aplicação ao solo do DLS e da CSS na ausência de palha, os valores de $\mathrm{N}$ mineral foram sempre positivos, o que indica ter ocorrido a mineralização líquida de N (Figura 3). Entretanto, na presença da palha de aveia, houve imobilização líquida de $\mathrm{N}$ até o final do experimento. Nos tratamentos com aplicação de DLS e CSS, sem a presença de palha,
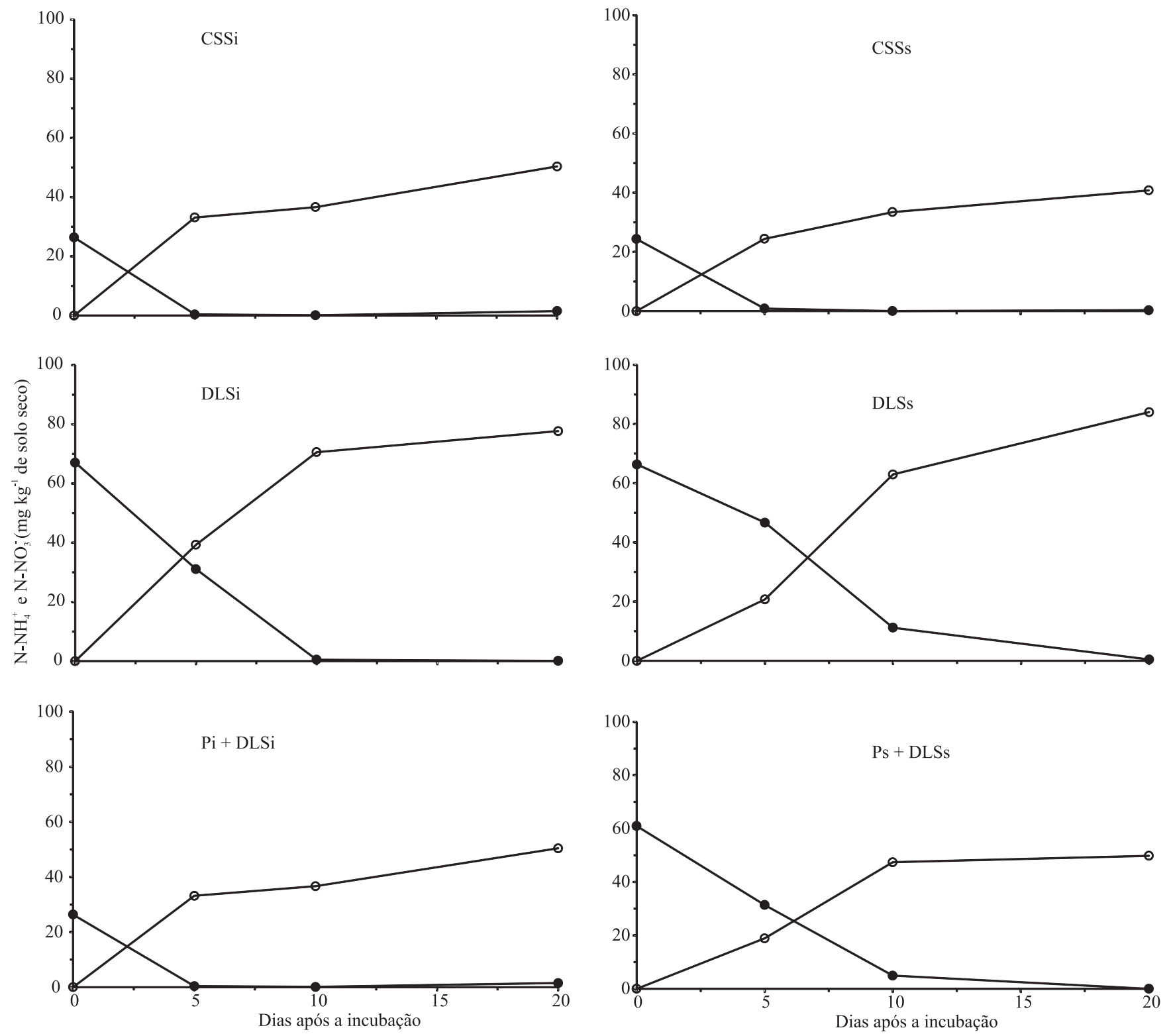
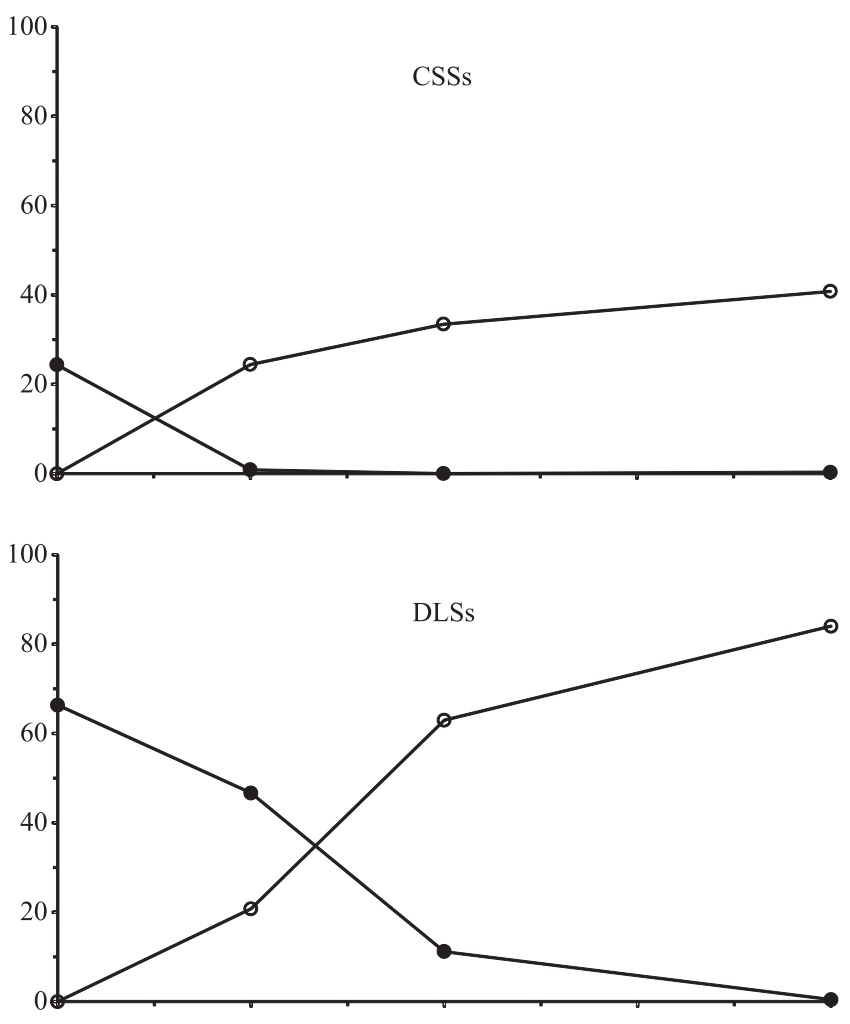

a mineralização líquida de $\mathrm{N}$ ocorreu durante os primeiros 30 e 50 dias, respectivamente, e não diferiu entre as modalidades de aplicação dos materiais orgânicos. Ao final da incubação, a mineralização líquida de $\mathrm{N}$ na média dos dois tratamentos com CSS foi de 43,2 $\mathrm{mg} \mathrm{kg}^{-1}$ de $\mathrm{N}$ no solo, o que corresponde a $14,6 \%$ do $\mathrm{N}$ orgânico adicionado ao solo com a

$\mathrm{N}-\mathrm{NH}_{4}^{+} \rightarrow-\mathrm{N}^{+} \mathrm{NO}_{3}^{-}$

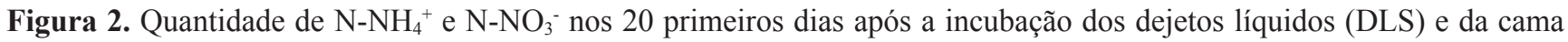
sobreposta (CSS) nos tratamentos com os materiais orgânicos aplicados na superfície (s) ou incorporados (i) ao solo, com e sem palha $(\mathrm{P})$. 
CSS. No mesmo período, a mineralização líquida média do DLS incorporado e em superfície foi de $21,6 \mathrm{mg} \mathrm{kg}^{-1}$ de $\mathrm{N}$ no solo, o que representa $34,9 \%$ de mineralização do $\mathrm{N}$ orgânico adicionado. Esse valor de mineralização, obtido para os tratamentos com DLS, é semelhante ao encontrado por Aita et al. (2012), também em condições de laboratório e à temperatura constante de $25^{\circ} \mathrm{C}$. No entanto, Morvan \& Nicolardot (2009), que trabalharam em condições de laboratório à temperatura constante de $15^{\circ} \mathrm{C}$, observaram mineralização de apenas $5,7 \%$ do $\mathrm{N}$ orgânico do DLS incorporado ao solo. Possivelmente, a menor mineralização do $\mathrm{N}$ obtida por estes autores,
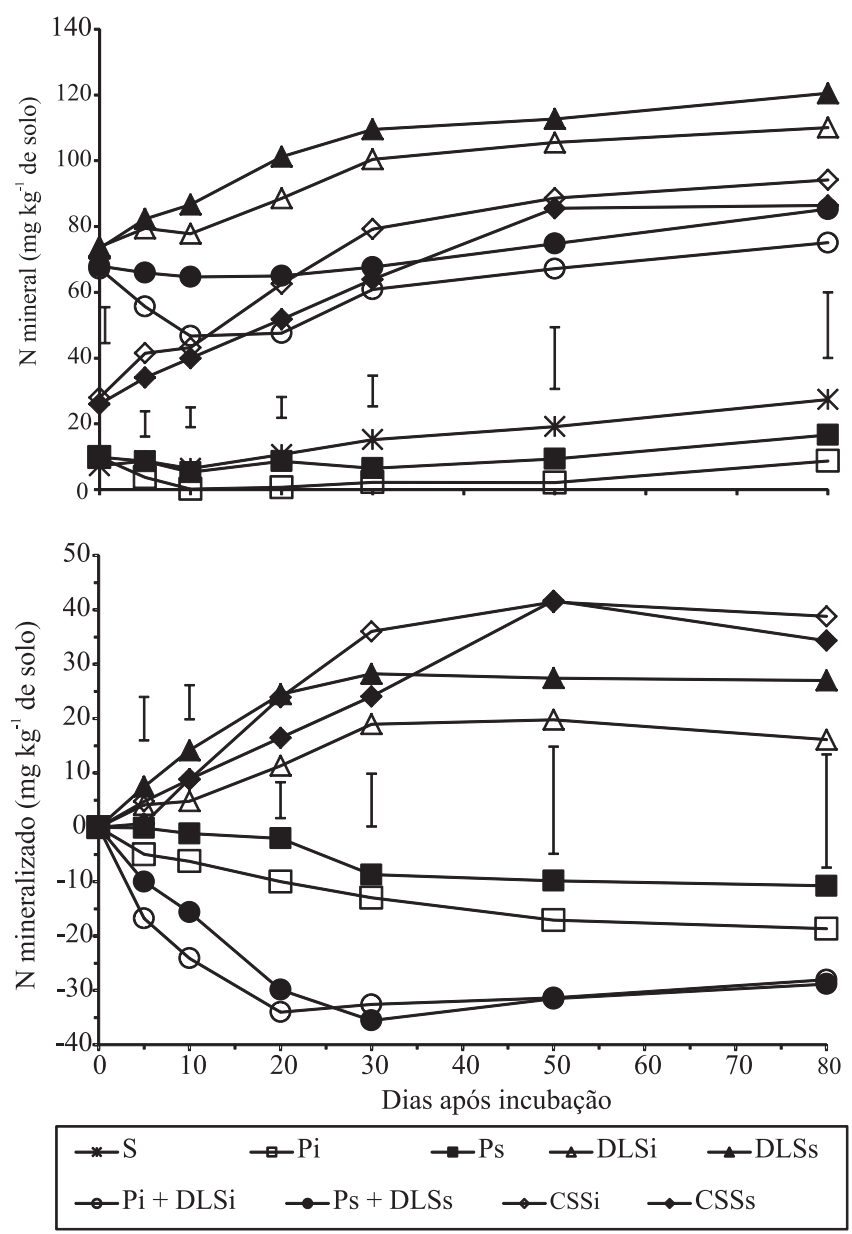

Figura 3. Quantidades de $\mathrm{N}$ mineral no solo (A) e de $\mathrm{N}$ mineralizado (B) nos tratamentos com adição de cama sobreposta (CSS), dejetos líquidos (DLS), palha de aveia (P) e palha + dejetos líquidos (P + DLS) na superfície (s) ou incorporados (i) ao solo (S). As barras verticais indicam a diferença mínima significativa pelo teste de Tukey, a 5\% de probabilidade. em comparação à encontrada no presente trabalho, resultou das diferenças nas características dos dejetos e da baixa temperatura de incubação utilizada.

A adição do DLS na superfície do solo levou a uma perda de $\mathrm{N}$ por volatilização de $\mathrm{NH}_{3}$ equivalente a $11 \%$ do $\mathrm{N}$ amoniacal aplicado, o que deveria resultar em quantidades inferiores de $\mathrm{N}$ mineral no solo, relativamente ao tratamento com incorporação do DLS ao solo; contudo, isso não foi observado.

Nos tratamentos com o uso exclusivo de palha, sem a aplicação de dejetos, houve diminuição nas quantidades de $\mathrm{N}$ mineral no solo desde a primeira avaliação, realizada cinco dias após a incubação, o que indica a ocorrência de imobilização líquida de $\mathrm{N}$ (Figura 3). Nesse período, a imobilização líquida de $\mathrm{N}$ foi de $0,1 \mathrm{mg} \mathrm{kg}^{-1}$ de $\mathrm{N}$ no solo, no tratamento com palha em superfície (Ps), e de 5,6 $\mathrm{mg} \mathrm{kg}^{-1}$ de $\mathrm{N}$ no solo, no tratamento com palha incorporada (Pi). Esses valores aumentaram até os 80 dias de incubação, tendo atingido valores de 10,8 e $18,7 \mathrm{mg} \mathrm{kg}^{-1}$ de $\mathrm{N}$ no solo, nos tratamentos $\mathrm{Ps}$ e $\mathrm{Pi}$, respectivamente. A maior imobilização de $\mathrm{N}$ observada com a incorporação da palha de aveia pode ser explicada em razão do aumento no contato do $\mathrm{C}$ da palha com os microrganismos do solo, o que favorece o acesso destes à água e aos nutrientes presentes no solo e aumenta a demanda microbiana de $\mathrm{N}$ para a decomposição da palha (Giacomini et al., 2008).

Quando se considerou apenas os tratamentos DLSs e Ps + DLSs, observou-se que a presença da palha de aveia causou redução significativa na quantidade de $\mathrm{N}$ mineral, desde a primeira amostragem realizada. Aos 80 dias, o solo do tratamento com palha apresentava $30 \%$ menos $\mathrm{N}$ mineral do que o solo do tratamento sem palha. A redução na quantidade de $\mathrm{N}$ mineral, provocada pela adição da palha, pode ser atribuída à imobilização microbiana de $\mathrm{N}$, em razão da alta relação $\mathrm{C} / \mathrm{N}$ da palha $(46,5 / 1)$, e à redução da mineralização da fração de $\mathrm{N}$ orgânico do DLS que permaneceu sobre a palha de aveia (Aita et al., 2012). Com a incorporação da palha, juntamente com o dejeto líquido de suínos (Pi + DLSi) também ocorreu diminuição significativa da quantidade de $\mathrm{N}$ mineral no solo, em comparação ao tratamento com incorporação somente dos dejetos (DLSi). Nos tratamentos com aplicação de N via DLS, a imobilização líquida de $\mathrm{N}$ atingiu valor máximo de $34,3 \mathrm{mg} \mathrm{kg}^{-1}$ de $\mathrm{N}$ no solo aos 20 dias, quando foram incorporados o DLS e os resíduos culturais da aveia, e de $36,5 \mathrm{mg} \mathrm{kg}^{-1}$ de $\mathrm{N}$ no solo aos 30 dias, quando 
o DLS foi adicionado sobre os resíduos culturais da aveia (Figura 3). Esses resultados são indicativos de que, na média desses dois tratamentos, a imobilização líquida de $\mathrm{N}$ atingiu $29 \%$ do $\mathrm{N}$ total aplicado com o DLS (Tabela 1). A imobilização de $\mathrm{N}$ durante a decomposição inicial de palhas de cereais em solo com a adição de $\mathrm{N}$ foi relatada por outros autores em estudos realizados em laboratório (Recous et al., 1995; Giacomini et al., 2009).

Os valores encontrados para a relação $\mathrm{mg}$ de $\mathrm{N}$ imobilizado/g de $\mathrm{C}$ adicionado foram de 11,4 e 12,2 para os tratamentos Pi + DLSi e Ps + DLSs, respectivamente, e de 3,6 e 6,0 para os tratamentos Pi e Ps, respectivamente (Figura $3 \mathrm{~B}$ ). Ao adicionar ao solo palha de milho com relação $\mathrm{C} / \mathrm{N}$ de 130 , Recous et al. (1995) observaram imobilização de $\mathrm{N}$ de $31 \mathrm{mg} \mathrm{g}^{-1}$ de C adicionado. A maior quantidade de $\mathrm{N}$ imobilizada por unidade de $\mathrm{C}$ adicionada encontrada por esses autores, em comparação à obtida no presente trabalho, pode ser atribuída à maior relação $\mathrm{C} / \mathrm{N}$ da palha utilizada, à maior disponibilidade de $\mathrm{N}$ no solo e ao fato de a palha ter sido finamente moída. No presente trabalho, os valores médios máximos da relação $\mathrm{N}$ imobilizado/C adicionado obtidos para os tratamentos Pi + DLSi e Ps + DLSs são indicativos da imobilização de $11,8 \mathrm{~kg}$ de $\mathrm{N}$ por $\mathrm{Mg}$ de $\mathrm{C}$ adicionado com a palha de aveia (4,7 $\mathrm{kg}$ de $\mathrm{N}$ por $\mathrm{Mg}$ de palha de aveia). Esse valor pode ser considerado como o potencial da aveia em favorecer a imobilização do $\mathrm{N}$ adicionado com o dejeto líquido de suínos.

\section{Conclusões}

1. A aplicação do dejeto líquido de suínos (DLS) na superfície do solo aumenta a volatilização de amônia, reduz a taxa de nitrificação do $\mathrm{N}$ amoniacal e não altera a taxa de mineralização do $\mathrm{N}$ orgânico, em comparação a sua incorporação ao solo.

2. O aporte de DLS sobre a palha de aveia, na superfície do solo, promove a imobilização de $\mathrm{N}$ sem reduzir a volatilização de amônia e a taxa de nitrificação do $\mathrm{N}$ amoniacal, quando comparado ao aporte apenas do DLS na superfície do solo.

3. A taxa de mineralização do $\mathrm{N}$ orgânico da cama sobreposta de suínos (CSS) é duas vezes menor do que a do DLS, independentemente da modalidade da aplicação, o que indica o menor potencial como fertilizante nitrogenado da CSS em comparação aos DLS.

\section{Referências}

AITA, C.; GIACOMINI, S.J.; HÜBNER, A.P. Nitrificação do nitrogênio amoniacal de dejetos líquidos de suínos em solo sob sistema de plantio direto. Pesquisa Agropecuária Brasileira, v.42, p.95-102, 2007. DOI: 10.1590/ S0100-204X2007000100013.

AITA, C.; RECOUS, S.; CARGNIN, R.H.O.; LUZ, L.P.; GIACOMINI, S.J. Impact on $\mathrm{C}$ and $\mathrm{N}$ dynamics of simultaneous application of pig slurry and wheat straw, as affected by their initial locations in soil. Biology and Fertility of Soils, v.48, p.633-642, 2012. DOI: 10.1007/s00374-011-0658-X.

CHANTIGNY, M.H.; ROCHETTE, P.; ANGERS, D.A.; MASSE, D.; CÔTÉ, D. Ammonia volatilization and selected soil characteristics following application of anaerobically digested pig slurry. Soil Science Society of American Journal, v.68, p.306-312, 2004. DOI: $10.2136 /$ sssaj2004.3060.

CORDOVIL, C.M.S.; VARENNES, A.; PINTO, R.; FERNANDES, R.C. Changes in mineral nitrogen, soil organic matter fractions and microbial community level physiological profiles after application of digested pig slurry and compost from municipal organic wastes to burned soils. Soil Biology and Biochemistry, v.43, p.845-852, 2011. DOI: 10.1016/j. soilbio.2010.12.023.

DENDOOVEN, L.; BONHOMME, E.; MERCKX, R.; VLASSAK, K. Injection of pig slurry and its effects on the dynamics of nitrogen and carbon in a loamy soil under laboratory conditions. Biology and Fertility of Soils, v.27, p.5-8, 1998. DOI: 10.1007/s003740050391.

GIACOMINI, S.J.; AITA, C. Cama sobreposta e dejetos líquidos de suínos como fonte de nitrogênio ao milho. Revista Brasileira de Ciência do Solo, v.32, p.195-205, 2008. DOI: 10.1590/ S0100-06832008000100019.

GIACOMINI, S.J.; AITA, C.; JANTALIA, C.P.; URQUIAGA, S.; SANTOS, G.F. dos. Imobilização do nitrogênio amoniacal de dejetos líquidos de suínos em plantio direto e preparo reduzido do solo. Revista Brasileira de Ciência do Solo, v.33, p.41-50, 2009. DOI: 10.1590/S0100-06832009000100005.

GIACOMINI, S.J.; AITA, C.; MIOLA, E.C.C.; RECOUS, S. Mineralização do carbono da palha de aveia e dejetos de suínos aplicados na superfície ou incorporados ao solo. Revista Brasileira de Ciência do Solo, v.32, p.2661-2668, 2008. DOI: 10.1590/ S0100-06832008000700008.

MORVAN, T.; NICOLARDOT, B. Role of organic fractions on $\mathrm{C}$ decomposition and $\mathrm{N}$ mineralization of animal wastes in soil. Biology and Fertility of Soils, v.45, p.477-486, 2009. DOI: 10.1007/s00374-009-0355-1.

NÖMMIK, H. The effect of pellet size on the ammonia loss from urea applied to forest. Plant and Soil, v.39, p.309-318, 1973. DOI: 10.1007/BF00014798.

OLIVEIRA, P.A.V. de. Comparaison des sistèmes d'élevage des porcs sur litière de sciure ou caillebotis intégral. 1999. 272p. Tese (Doutorado) - Ecole Nationale Supérieure Agronomique de Rennes, Rennes. 
PORT, O.; AITA, C.; GIACOMINI, S.J. Perda de nitrogênio por volatilização de amônia com o uso de dejetos de suínos em plantio direto. Pesquisa Agropecuária Brasileira, v.38, p.857-865, 2003. DOI: 10.1590/S0100-204X2003000700010.

RECOUS, S.; ROBIN, D.; DARWIS, D.; MARY, B. Soil inorganic N availability: effect on maize residue decomposition. Soil Biology and Biochemistry, v.27, p.1529-1538, 1995. DOI: 10.1016/0038-0717(95)00096-W.

SÁNCHEZ, M.; GONZÁLEZ, J.L. The fertilizer value of pig slurry. I. Values depending on the type of operation. Bioresource Technology, v.96, p.1117-1123, 2005. DOI: 10.1016/j. biortech.2004.10.002.
SANTOS, H.G. dos; JACOMINE, P.K.T.; ANJOS, L.H.C. dos; OLIVEIRA, V.A.; OLIVEIRA, J.B. de; COELHO, M.R.; LUMBRERAS, J.F.; CUNHA, T.J.F. (Ed.). Sistema brasileiro de classificação de solos. 2.ed. Rio de Janeiro: Embrapa Solos, 2006. $306 \mathrm{p}$.

TEDESCO, M.J.; GIANELLO, C.; BISSANI, C.A.; BOHNEN, H.; VOLKWEISS, S.J. Análises de solo, plantas e outros materiais. 2.ed. Porto Alegre: Universidade Federal do Rio Grande do Sul, 1995. 174p. (UFRGS. Boletim técnico, 5).

TIQUIA, S.M.; RICHARD, T.L.; HONEYMAN, M.S. Carbon, nutrient, and mass loss during composting. Nutrient Cycling in Agroecosystems, v.62, p.15-24, 2002. DOI: 10.1023/A:1015137922816.

Recebido em 9 de agosto de 2012 e aprovado em 30 de janeiro de 2013 